\title{
Implementation of Polyvynil-Alcohol Mixed With Nano-Particles as a Near Representation of Biological Tissue: Ultrasonic and Photo- Thermal Study
}

\author{
R. M. Quispe-Siccha* ${ }^{1}$, B. Reyes-Ramírez ${ }^{1}$, C. García-Segundo ${ }^{1}$, \\ N. Hevia-Montiel ${ }^{4}$, F. Arámbula-Cosío ${ }^{1}$, R. Sato-Berrú ${ }^{1}$, J. O. Flores-Flores ${ }^{1}$ \\ ${ }^{1}$ Centro de Ciencias Aplicadas y Desarrollo Tecnológico, \\ Universidad Nacional Autónoma de México, \\ Circuito Exterior Universitario. Apdo. Postal (P.O. Box) 70-186. \\ Coyoacán 04510. México, D.F. \\ ${ }^{4}$ Departamento de Ciencias de la Computación, \\ Instituto de Investigaciones en Matemáticas Aplicadas y en Sistemas \\ Universidad Nacional Autónoma de México (UNAM) Apartado Postal 20-726, \\ Ciudad Universitaria, C.P. 04510. México, D.F. \\ *rosa.quispe@gmail.com
}

\author{
1ST INTERNATIONAL \\ CONGRESS ON \\ INSTRUMENTATION AND \\ APPLIED SCIENCES
}

\begin{abstract}
We present experimental results related to the manufacturing of phantoms based on polyvinyl-alcohol (PVA) mixed with $\mathrm{SiO}_{2}$, graphite and $\mathrm{Ag}$ nanoparticles. These phantoms are dummy representations of the human tissue of the breast gland. We focus our attention on the representation of the optical and the mechanical properties of the actual biological healthy tissue and of that representing a hidden cancer tumor (lesion). The quality of the samples is tested by ultrasonic imaging and laser induced photothermal (PT) detection technique. From the former test, the mechanical contrast that one can achieve is apparent. Instead, the laser detection technique makes it possible to determine that the optical contrast is achievable. For the laser induced PT method we register the so-called photothermal amplitudes, rather known as photoacoustic (PA) signals. These are the ultimate expression of laser-induced bursts of photothermal processes, whose engine is the fraction of the optically absorbed energy that decays through nonradiative channels. In our case we look for the conditions at which the PT phenomena are produced by a hidden dummy-tumor alone; meaning that the output signals are nearly free from substantial contributions from the bulk of the phantom. This is so as to mimic the actual mechanical and optical absorption performance of breast tissue with an inner cancer tumor. From the ultrasound images one can see how apparent the resemblance with the actual human tissue is.
\end{abstract}

Keywords: Polyvinyl-alcohol, nanoparticles, photothermal, ultrasound, phantom.

\section{RESUMEN}

Presentamos resultados experimentales sobre la fabricación de fantasmas a base de alcohol polivinílico (PVA) mezclado con nano-partículas de $\mathrm{SiO}_{2}$, grafito y Ag. Estos fantasmas son una representación artificial del tejido humano de la glándula mamaria. Nos enfocamos en la representación de las propiedades ópticas y mecánicas de tejidos sanos y de tejidos de lesiones o anomalías internas. Esto en clara representación de un tumor cancerígeno. La calidad de las muestras se analizan mediante imágenes de ultrasonido y mediante detección de procesos fototérmicos (FT) inducidos por láser. El primer método de análisis nos permite verificar de manera inmediata el contraste mecánico que se obtiene de las muestras. La detección FT permite mostrar el contraste óptico que se logra. Esto se hace mediante el registro de la amplitud de las perturbaciones foto-térmicas inducidas por pulsos láser, también conocidas como señales fotoacústicas. Estas son las expresiones más representativas de los fenómenos fototérmicos, inducidos por la fracción de la energía óptica absorbida que decae a través de procesos no-radiativos. En nuestro caso buscamos las condiciones en las cuales los fenómenos fototérmicos son generados únicamente por la lesión oculta en el maniquí, sin contribución sustancial de la masa del fantasma. De esta manera, logramos representar el desempeño óptico y mecánico del tejido de seno con un tumor en su interior. De las imágenes de ultrasonido, es evidente la semejanza con el tejido humano real.

\section{Introduction}

Over the last years there has been a large amount of efforts dedicated to the development of novel and non-invasive imagenology technologies, in particular to those focused on breast cancer diagnosis. The general aim is to achieve an alternative imaging method of diagnostic respect to the radiological imaging methods. Particularly, the use of near-infrared (NIR) radiation, from laser 
pulsed systems, has proven to be a cost-effective method [1, 2]. This is as result of the fact that the bulk tissue is optically non-absorbent at the nearinfrared bandwidth and instead is rather optically diffusive. Note that within the bulk tissue, only vascular material absorbs light at these wavelengths. This characteristic makes it possible to observe a large optical contrast between healthy bulk tissue and a cancer tumor, which is vascular-tissue largely dense. The vascular absorption would partially decay via non-radiative channels, prompting laser-induced ultrasound signals, termed as photoacoustic (PA) or photothermal (PT) [1] perturbations. Among the advantages of these techniques it is that one can get images free from diffuse NIR radiation, they are non-invasive and, the output signals can possess a large signal-to-noise ratio [2]. Furthermore, these techniques can be integrated to medical protocols on computer-assisted biopsy [3] and even to the so-called optical biopsy $[4,5]$.

On the other hand, the advancement in nanotechnology has hadmade a direct impact in the development of methodologies for cancer diagnosis at the micro- and nano-scale. Up to date, one of the most common uses of nanoparticles combined with laser pulses interaction are these methodologies for diagnostic and for remission of a cancer tumor. They are based on the fact that in the interaction with laser pulses there are two main processes take place $[5,6]:$ 1) Once the laser energy is absorbed, it induces intermittent particle thermal expansion, and then the generation of PT perturbations traveling all through according with the thermoelastic properties of the tissue [7]. In this way, one can identify the place and dimensions of the tumor; 2) The other process is that in which the excess of heat results in the burning of the tissue in the vicinity of the nanoparticles; the expectation is that in this way in somehow one can help to the remission of a tumor [8].

Before reaching the stage of these advanced applications, including the use of biological models, one needs to produce testings in the shape of controlled laboratory -experiments. These are made with the aid of dummy samples (phantoms). Among the most frequent types of phantoms are those manufactured with agar, polyester, epoxy -resins and natural rubber [9]. In each case, the model is tailormade for a specific purpose since the optical, the mass density, the elasto-mechanical and the thermal properties are not easy to manipulate [10]. More recently, the Polyvinyl-alcohol (PVA) has been suggested to be as better material to represent some of these properties at once [11]. In the present report we verify this assertion, and we extend the range of manipulation by mixing up the PVA with nanoparticles of specific kinds at specific dosages. The physical functionality of the PVA phantoms is such that we can use these as a likely representation of the breast tissue for PT studies and for ultrasound imaging.

Thus, as bottom-line, we combine PVA with nanoparticles to build up dummy representations of human tissue. We use one kind of nanoparticle to represent the bulk's optical scattering and a given mass density. Then, we use a second kind of nanoparticle to represent graded optical scattering, graded optical absorption and to modify the mass density, which is required to be slightly different from the bulk. With these experiments, we are also looking for ways to better understand the underpinning phenomenology involved in the theoretical models describing the generation and transport of energy in complex systems. In overall, the process must provide us with a highly reproducible and acceptable costeffective solution.

\section{Experiment}

For means of better understanding of the involved phenomena, we splitted the experiment in two stages. In the first stage, we deal with colloidal samples, since from the experimental point of view these are a good enough first approach to the representation of the phantom in a near-complex mode. Furthermore, it is advisable to recognize the nanoparticles response to the laser stimulus, prior to be inserted within the phantom; which is already a complex medium. On the second stage, we deal with the actual phantoms, profiting from the learning we earn from the first stage.

\subsection{Experiments with colloids}

The colloid samples are home-made nanoparticles [12] suspended in a liquid matrix, at a known particle concentration per volume unit. For this purpose, we use nanoparticles of $\mathrm{SiO}_{2}$ of $450 \pm 40 \mathrm{~nm}$ diameter, graphite of $60 \pm 10 \mathrm{~nm}$ diameter and $\mathrm{Ag}$ of mixed diameter sizes (ranging from 5 to near $100 \mathrm{~nm}$ diameter). The particle size is experimentally determined using a commercial Z-Sizer (NANO-ZS90, Malvern Inst.) system. 


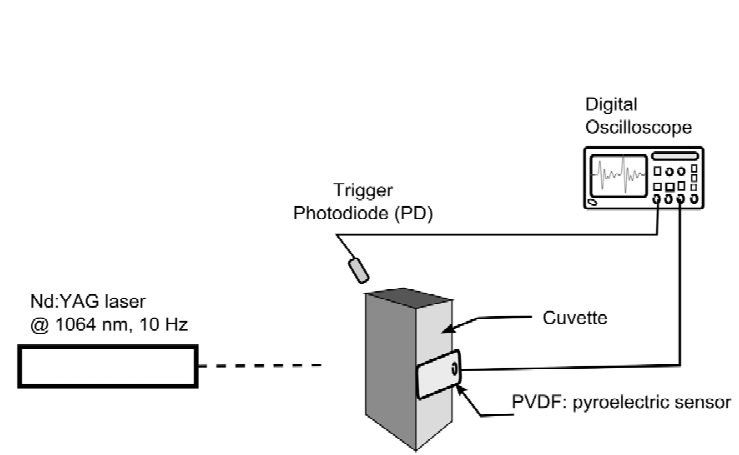

a)

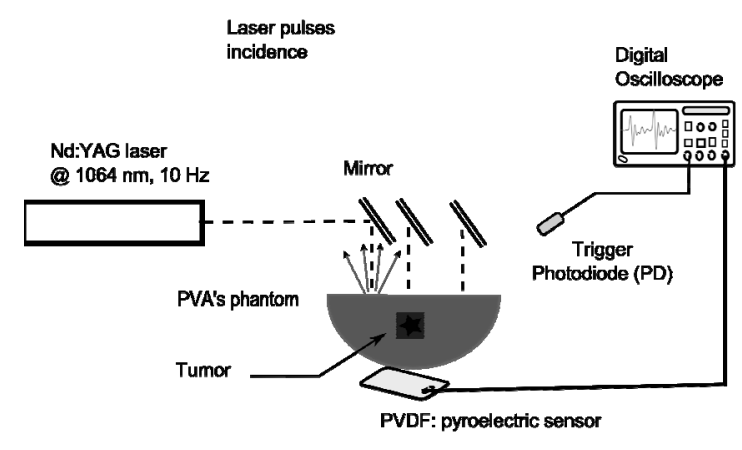

b)

Figure 1. (a) Experimental set up for laser testing of colloids, and (b) for the PVA phantoms mixed with nanoparticles and the embedded tumor. The PVDF film is in physical contact with the phantom through a buffer gel aiming to match its mechanical impedances. Notice that in both experimental set ups we use the so-called transmittance configuration.

In Figure (1a.), we display a cartoon-sketch of the experimental set up used to analyze the colloidal samples. In these experiments the starting point is to have $3 \mathrm{ml}$ of colloid made of a high concentration of nanoparticles suspended in distilled water, in a quartz spectroscopic cuvette of 10x10x40 mm (Q100, Hellma Cells). This sample is pumped with laser pulses triggered from a Nd:YAG laser (Brio-120, Quantel), impinging perpendicular to one of the windows of the cuvette. The laser emission wavelength is set at $1064 \mathrm{~nm}$, having pulses emitted at $10 \mathrm{~Hz}$ rate, whose pulse width is of 5.4 ns (FWHM) and fluency of 0.32 $\mathrm{J} / \mathrm{cm}^{2}$. This process induces laser light absorption, from which a portion is photothermally transformed as a mechanical burst of energy, building up the so-called PA signal. This travels at a sound speed of $1500 \mathrm{~m} / \mathrm{s}$, carrying ultrasonic frequencies, emerging at the opposite end with respect to the point where the laser-beam interaction takes place. A portion of this perturbation is read out with a commercial pyroelectric sensor, attached to the window of a cuvette whose area is perpendicular to the laser incidence direction (see Figure 1a). Then the sensor output is matched to a $50 \mathrm{ohm}$ terminated digital-oscilloscope, synchronized with the instant at which each laser pulse is shot through a $1.0 \mathrm{~ns}$ rise-time photo-diode (PD). A single experimental measurement is the result of the average of at least 100 events (pulses), which causes the measurement error to drastically go down to less than one percent of the peak signal and, thus, to be out of scale to be displayed. Once this measurement is over, this initial $3 \mathrm{ml}$ sample is cleanly removed from the cuvette and then, in a separate deposit, it is mixed with an extra $1 \mathrm{ml}$ of pure distilled water. From the resulting concentration, we take $3 \mathrm{ml}$ of colloid, which is dropped into the cleaned cuvette. Then this new sample is also pumped by the laser pulses, repeating then the measurement process of the previous concentration. The process of diluting the sample and then laser testing it is repeated over and over until the PA signals from the colloid and from distilled water (free from nanoparticles) have no significant amplitude and shape differences. The initial number of nanoparticles in the $\mathrm{SiO}_{2}$ colloid is of $3.87 \times 10^{11}$ per $\mathrm{ml}$, which corresponds to a volume fraction of $0.017(1.7 \%)$. In average, the PA signal propagates through an average effective distance of $2 \mathrm{~cm}$. This being calculated from the time of arrival of the PA signal and the colloid sound speed.

The same procedure is repeated for each kind of particles we report here. As result of these measurements, we obtained curves of PA amplitude as a function of the particle concentration per volume unit. In the next section we present these results. At this stage, the main outcome of the measurements is to determine the nanoparticle concentration at which we get the maximum PA-amplitude. 


\subsection{Experiment with phantom}

From the previous experiment, we take the estimates of particle concentration to be used as the basis for preparing the mix of PVA with nanoparticles, and thus to get the dummies representing the breast bulk and tumors. Thus, the criteria to determine which nanoparticle should be used for the dummy tissue and which one for the dummy lesion (tumor), depends on the chemical stability of the nanoparticle, the optical scattering, the optical absorption and the miscibility. The dummy representing the bulk is mixed with nanoparticles of larger optical scattering compared with its optical absorption. From this criterion, it turns out that the $\mathrm{TiO}_{2}$ nanoparticles are better scatterers, having $8.02 \mathrm{~cm}^{-1}$ scattering coefficient, and near zero optical absorption at $1064 \mathrm{~nm}$. Instead, $\mathrm{SiO}_{2}$ in PVA produces an absorption coefficient of $1.81 \mathrm{~cm}^{-1}$ that is large enough to represent a graded optical contrast.

The procedure for the preparation of the PVA phantom is as the state of the art reported in the literature [10], with the difference that we include the nanoparticles. Distilled water is heated up to the boiling point temperature $\left(\sim 94{ }^{\circ} \mathrm{C}\right)$, and then the PVA powder is added at a concentration of $8 \%$ per volume of water, taking care that the mix is steered slow and continuously. The mixture is kept at boiling temperature for as long as it takes the powder to dissolve in the water. After some minutes we added the $\mathrm{TiO}_{2}$ nanoparticles, keeping the slow and continuous steering of the mixture. The final product is left to cool down at room temperature. Once is cool enough, this is brought to near 24 hour cycles of freezing and unfreezing: 12 hours at $-20{ }^{\circ} \mathrm{C}$ and 12 hours at room temperature. In this way, after 5 to 8 cycles, the PVA mix hardens, achieving a consistency as that of natural rubber, with the advantage of having porosity and a certain level of moisture. Throughout the hardening process of the sample made with PVA with $\mathrm{TiO}_{2}$ nanoparticles, we insert a piece of already hardened PVA with $\mathrm{SiO}_{2}$ nanoparticles. In this way, we set up a sample containing graded properties of the optical scattering, optical absorption, the mass density differences and the embedded moisture. The result is a dummy that mimics the optical and mechanical properties of human breast tissue with abnormalities. Once the PVA phantom is ready, we insert it in the laser testing set-up as the carton displayed in Figure (1b).

\section{Results and discussion}

\subsection{Colloids}

Other tests carried out with optically absorbent nanoparticles, apart from $\mathrm{SiO}_{2}$, were those on graphite and $\mathrm{Ag}$. Because of the optical window at which the photothermal tomography is working (900 to near $2000 \mathrm{~nm}$ ), we restrict ourselves from working out the responses from these particles within the near infrared (NIR) range. The test we performed is as we described in the previous section.

We use samples of $\mathrm{SiO}_{2}$ colloid to display the overall performance of the PA amplitude as a function of their concentration. The results are presented in Figure (2a), where it can be seen that when the colloid is diluted, the PA amplitude increases with respect to the initial value. We assume that it is so because the effective volume of interaction increases up to a given value. However, as the colloid is further diluted, although the laser light has deeper penetration within the colloid, one reaches a maximum effective interaction volume beyond which this effective interaction volume decreases. It means that the actual number of nanoparticles available for interaction has been reduced, and thus the PA amplitude will also decrease. The same figure (2b) shows that the speed of propagation of the PA signal within the colloid follows a performance as that exhibited by the PA amplitude. In our interpretation, this is because the actual meanfree-path of the PA perturbation changes with the particle concentration.

The output signals from graphite and Ag colloids, are displayed in $\mathrm{F}$ the figure (3). Besides the small amplitude, these are comparatively more complex than those for $\mathrm{SiO}_{2}$. Therefore, because of the larger amplitude of the output signal and its simpler shape, it seems to be rather clear that the $\mathrm{SiO}_{2}$ nanoparticles are the best choice for mixing up with the PVA, and thus for obtaining the phantoms for the dummy representation of the optical absorption and the optical scattering of a tumor. 

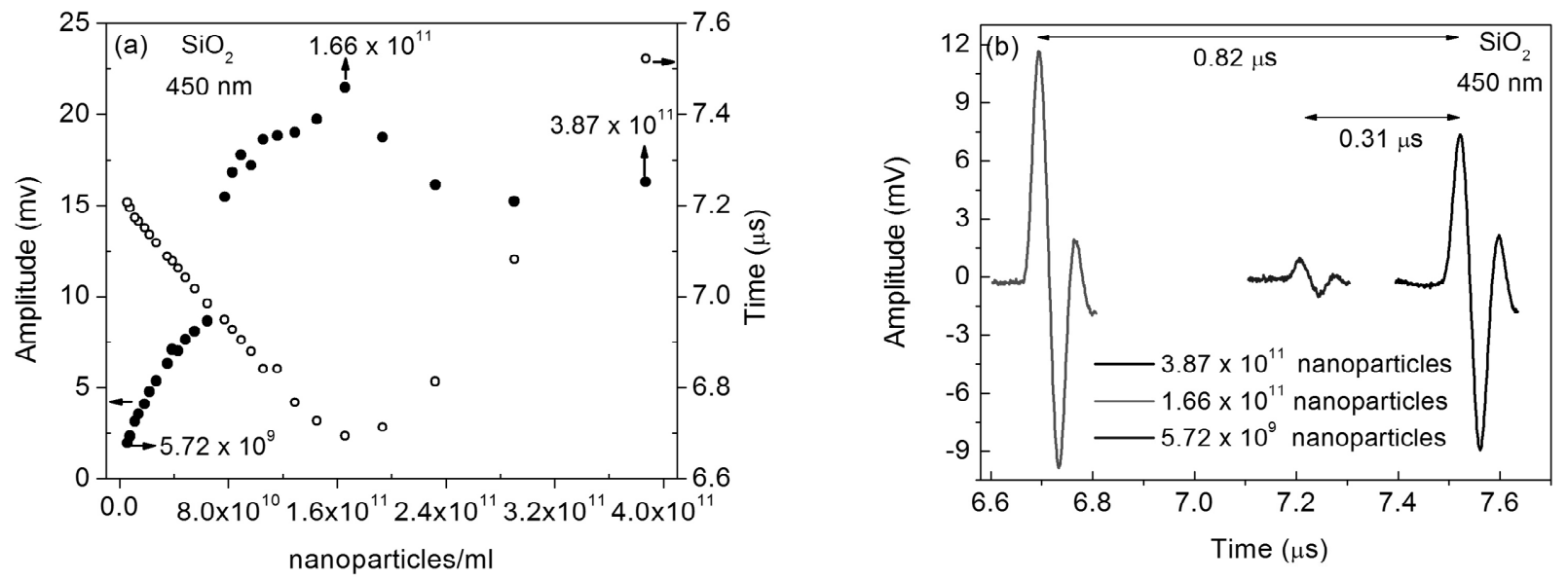

Figure 2. Colloid sample of $\mathrm{SiO}_{2}$ in distilled water. The curves in (a) are the PA amplitude (black dots) and the time of arrival (white dots) of the signal with respect to the laser trigger; both as a function of the number of nanoparticles per volume unit. In (b) we display the actual PA signal output for different times of arrival. The right-hand side curve belongs to the sample at concentration of $3.87 \times 10^{11} \mathrm{particles} / \mathrm{ml}$, the middle curve is that for $5.72 \times 10^{9}$ particles $/ \mathrm{ml}$, and the left-hand side curve is that of $1.66 \times 10^{11}$ particles/ml and produces the largest PA amplitude and propagation speed.
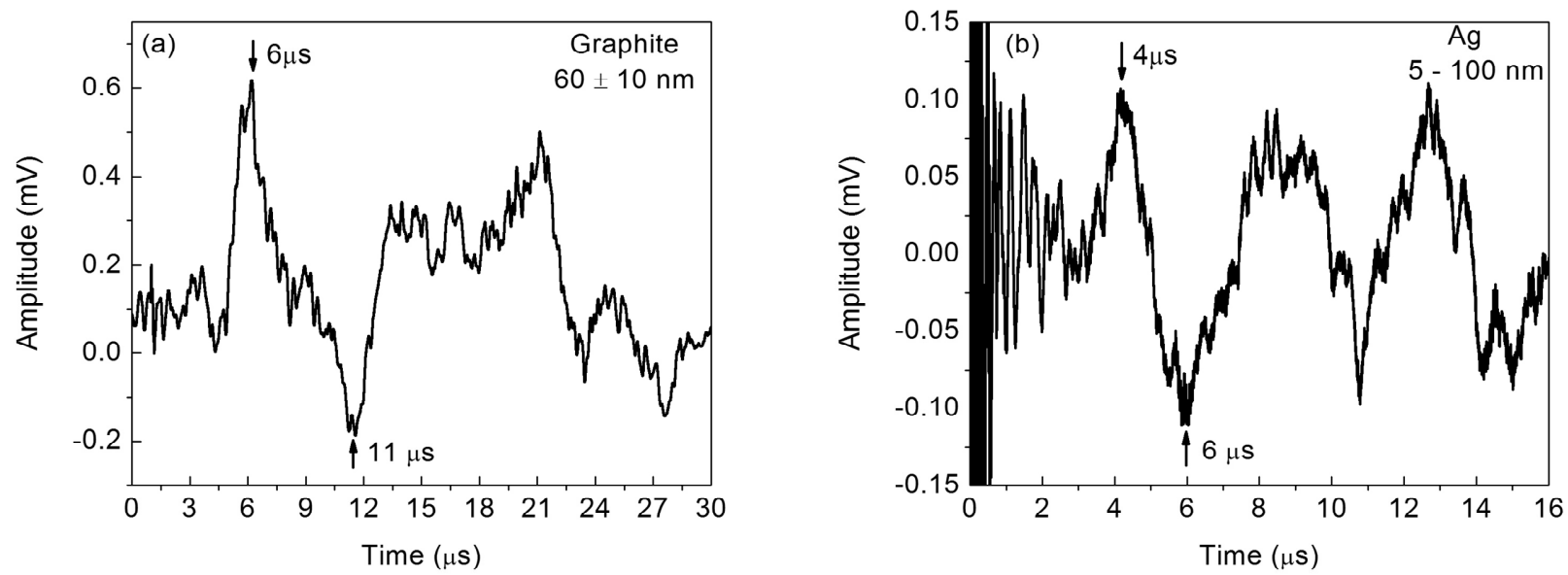

Figure 3. The photo-thermal signal read out from graphite (a) and from $\mathrm{Ag}$ (b).

The particle sizes are $60 \pm 10 \mathrm{~nm}$ and wide distribution from 5 to $100 \mathrm{~nm}$, respectively. 
One important feature of the PA signals emerging from the colloid is the characteristic shape they have, which shall depend on the nanoparticles composition. From reiterative repetitions of these experiments, we verified that these associated signal shapes, can be reproduced over and over. Thus, these shapes can be used as a fingerprint of the PA response of the phantoms. This means that, once the lesion is within the phantom bulk, one can distinguish the PA signal of the phantombulk can be distinguished with respect to that from the dummy lesion. Furthermore, we could distinguish which kind of nanoparticles was used for manufacturing the dummy lesion which. It means that with our method we can add a specificity factor.

For $\mathrm{SiO}_{2}$, we obtain that the maximum of $\mathrm{PA}$ amplitude is of $(21.5 \mathrm{mV})$ for samples of particles of mean diameter of $450 \mathrm{~nm}$. Instead, for independent samples of graphite (mean particle diameter $\sim 60 \pm 10 \mathrm{~nm}$ ) and $\mathrm{Ag}$ (wide-spread diameter, 5 to $100 \mathrm{~nm}$ ), at $0.4 \%$ volume concentration, we obtained PA amplitudes of 0.80 $\mathrm{mV}$ and $0.20 \mathrm{mV}$, respectively.

\subsection{Phantoms}

The bulk of the phantoms is made using commercial natural size breast molds. Special care was taken to locate the dummy lesion (PVA with $\mathrm{SiO}_{2}$ nanoparticles ) by the axis of the breast cone made of PVA mixed with $\mathrm{TiO}_{2}$. The final phantom as we obtained is shown in Figure (4a). The PT experiments were carried out using the set up displayed in Figure (1b). We recovered the signal at three different directions: two positions off the axis and one position on the axis of the breast cone. The signal outputs, as displayed in Figure (4b) are those registered from the oscilloscope, being the upper and lower curves for the off-axis incidence and the middle one for the on-axis incidence. The equivalent curves for phantoms with graphite and Ag mixed in the dummy lesion are displayed in Figure (5) with an organization of curves as that in Figure (4). Notice that the signal registered from off-axis positions has no-visible PA output content, while that on-axis (lesion position) has the distinctive PA mark observed in colloids.

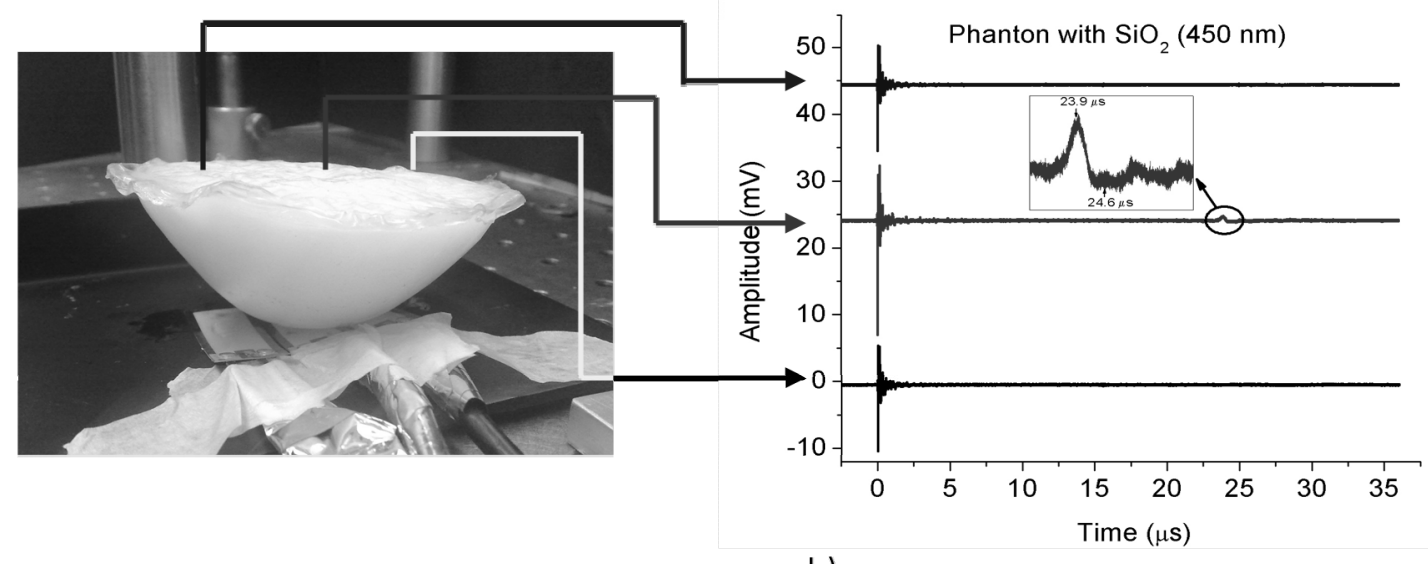

a)

b)

Figure 4. (a) The PVA phantom with lesion made of PVA with $\mathrm{SiO}_{2}$ nanoparticles.

(b) The photo-thermal signals at 3 different locations of the phantom. Side ones, lesion free, middle one is to target the dummy lesion. 
The peak of amplitude from the dummy lesion based on $\mathrm{SiO}_{2}$, appears at $23.9 \mu$ s clocked from the instant at which each laser pulse impinges on the phantom's surface using a fast photodiode (0.5) ns rise time; see Figure (1b) for general guidance. For the number of freezing/unfreezing cycles, we use the value of $1550 \mathrm{~m} / \mathrm{s}$ as speed of sound within the PVA, matching with the literature values [11]. The dummy lesion was set at $37 \mathrm{~mm}$ depth with respect to the point of detection, set at the lower point of the breast cone (see Figure 4a). From the time difference between the first maximum and minimum, the burst of signal, we estimate the heat source extension: being $1.1 \mathrm{~mm}$ for the dummy lesion based on $\mathrm{SiO}_{2}$. It should be noted that the heat source dimensions is not necessarily associated to the laser beam diameter.

As a given pulse of light impinges on the sample, a fraction of the light is back-scattered and a larger portion will propagate within the phantom bulk. Due to the bulk's optical scattering properties, the beam shape is widely scattered within this bulk. Therefore, only a small portion of the initial fluency shall arrive to the dummy lesion, producing the PA signal. Then the PA signal travels across a portion of the dummy lesion and across the remaining portion of phantom's bulk. The structure of this lesion and phantom is somehow a disordered structure and altogether with the nanoparticles will exhibit resistance to the free propagation of the PA

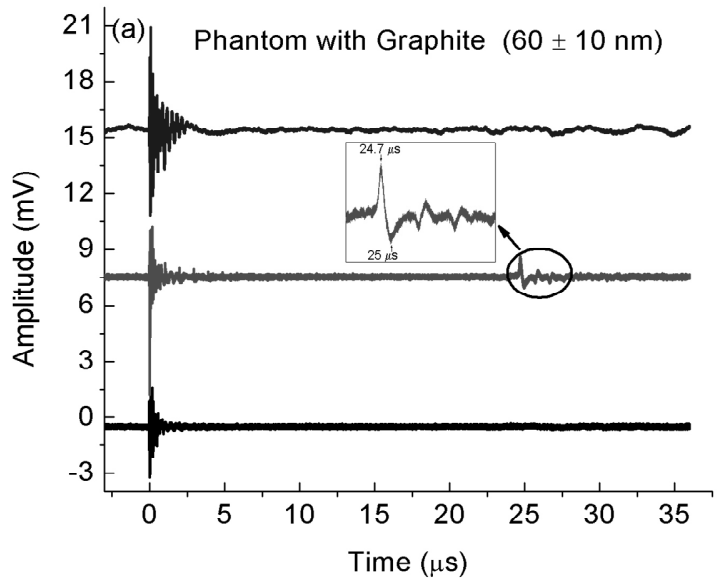

signal. As result of this condition, we lose a fraction of the PA amplitude. Thus the final output signal is only a portion of the original PA signal. The precision we can achieve will depend very much on the depth of propagation of the light, the magnitude of the PA signal, and the degree of complexity of the sample. This complexity condition is quite similar to that occurring in natural tissue [5].

On the other hand, from the dummy breast with the lesion made with graphite and $\mathrm{Ag}$, respectively, the dummy lesion is at $37 \mathrm{~mm}$ depth and at $40 \mathrm{~mm}$ with respect to the point of sensing the signal (see Figure 5). The heat source diameter was estimated to be 0.5 and $0.3 \mathrm{~mm}$, respectively.

The PA amplitudes for $\mathrm{SiO}_{2}$, graphite and $\mathrm{Ag}$, using $40 \mathrm{~mJ}$ of laser energy on a $4 \mathrm{~mm}$ crossbeam diameter is $0.89 \mathrm{mV}, 1.61 \mathrm{mV}$ and $0.91 \mathrm{mV}$, respectively.

This time, it can be observed that the dummy with graphite lesion produces the larger PA amplitude output. The difference of result between the colloids output and the phantom is because the graphite in the water matrix is barely suspended. Its weight makes it precipitate in less than 5 minutes and, therefore, the control on the exact number of scatterers or rather the optical absorption cross section changes relatively too fast. As result, the PA output amplitude fluctuates

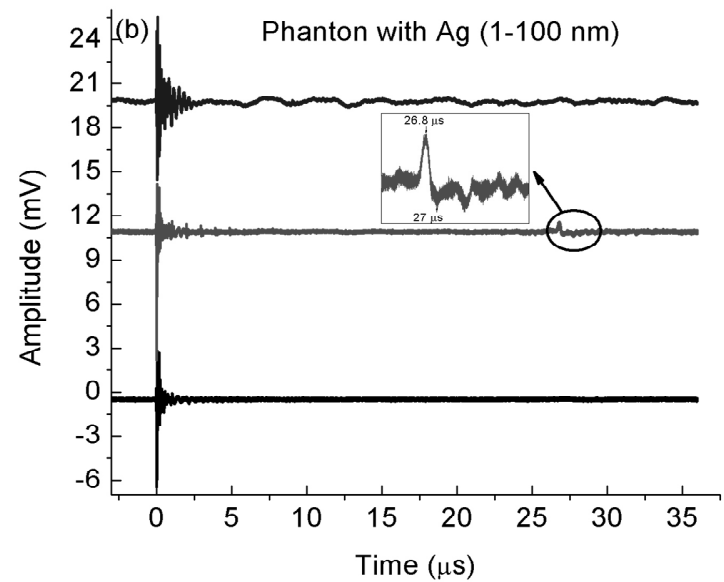

Figure 5. The PA signal output from the phantom of PVA with graphite (a) and Ag (b) nanoparticles. The signals are as displayed on the oscilloscope. 
in an uncontrolled way. Instead when the graphite is inserted in PVA, it is set with static random distribution, and thus the optical absorption cross section remains as a static parameter too.

Among the main outcomes is that the actual PA response from each phantom has a different shape and amplitude. The main features identified as ruling properties are the chemical and physical constitution of the embedded nanoparticles, their spatial distribution (volumetric density), and their mean diameter. Hence, we have now a methodology to grade and handle the optical and mechanical density of the phantoms and of the embedded lesion. To verify the estimates, ultrasonic images were obtained from each of the phantoms with the embedded lesion. For that purpose, a commercial ultrasound (US) system (Bscan, Polaris Northen Digital) was employed, of the type used in medical ultrasound imagenology. This process was applied in the case of the three types of phantoms mentioned above which were produced in the same batch as to guarantee that each PVA bulk has the same physical conditions. As result, it was verified that indeed the nanostructured materials can simulate the lesions (tumors), and these provide a very good realistic ultrasound image of a tumor.
The registered images are displayed in Figure (6). These were obtained from a near conical phantom (see Figure $4 \mathrm{a}$ ) of $12 \mathrm{~cm}$ diameter and $8 \mathrm{~cm}$ high. The inserted lesion is a near cylindrical object of $1.5 \mathrm{~cm}$ diameter and $1.0 \mathrm{~cm}$ high. The images on the left-hand side column correspond to USscanning of phantom volume without a dummy lesion. These were taken at near 45 degrees with respect to the vertical. The images on the righthand side column display the lesion (taken at the upper portion of the breast cone). Figures (6a) and $(6 \mathrm{~b})$ correspond to the phantom with a lesion based on $\mathrm{SiO}_{2}$ nanoparticles. Figures (7c) and (6d) are from that based on graphite, and Figures (6e) and (6f) correspond to the Ag case. In each case, the granularity in the bulk of the phantom produces a remarkable resemblance to the granularity displayed in US images from actual breast tissue [13]. Indeed, the image contrast between the bulk and the lesion is inverted with respect to a real breast with a cancer tumor; nevertheless the mechanical contrast we achieve in the samples is clear and in mismatch with optical contrast registered with the PA measurements (these displayed on the figures 4 and 5). For the purposes of the present testing, such results are good enough and are the most important features we were targeting. a)

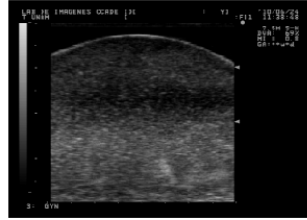

c)

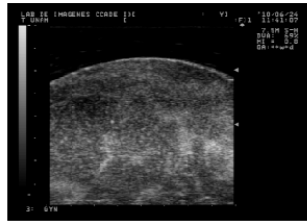

e)

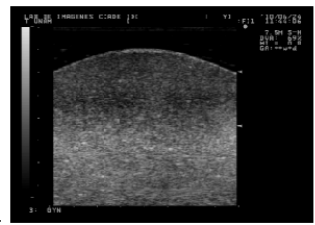

b)

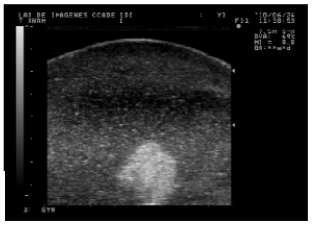

d)

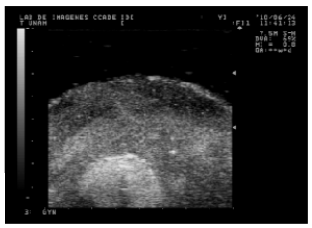

f)

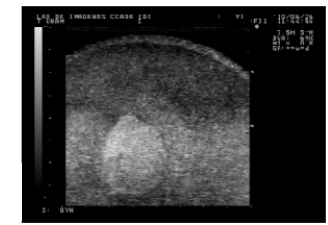

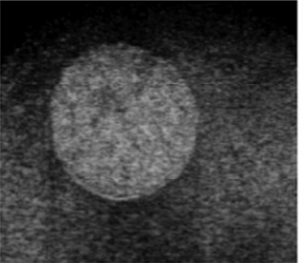

g)

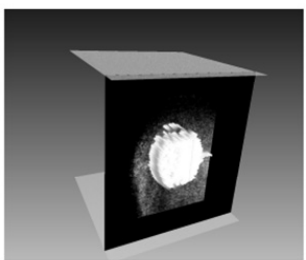

Figure 6. Ultrasound images from phantoms with embedded dummy lesions, the dimensions are as indicated in the main text. From (a) to (f) display volume portions with and without lesion presence, for the three kinds of phantomss we describes here and $(\mathrm{g})$ display the image reconstruction. Details about meaning and correspondence is in the main text. 
The quality of the phantoms is such that it permits the manipulation of the image for the purpose of segmentation and image post-processing; thus, the quality of the US-images we get out of them is such that is possible to use these images to implement algorithms on image processing reconstruction and segmentation, as can be seen from the example we display Figure $(6 \mathrm{~g})$ [4]. The reconstruction of the volume was performed obtaining images including the 3D position and translation of the ultrasound probe. With this data, a transformation matrix was calculated for each image and then all the pixels in the image were transformed in order to place every image according to the position and translation obtained.

\section{Conclusions}

From the comparative experimental analysis we present here we can assert that the manufacture of phantoms made of PVA mixed with nanoparticles represent a powerful alternative for manufacturing lab models that represent human tissue. We verify that we can maneuver to gain a good enough control on the kind of optical (PA measurements) and mechanical (US images) contrast as required for lab testing on tomography and other computer assisted procedures. This is possible by just paying special attention to the nature of nanoparticles to be used. We also verify that with the method we describe here, the PVA based phantoms can be washed and stabilized by keeping them in a covered and clean moist environment, in addition to the fact that the manufacturing process is highly reproducible and the samples are stable. This condition can be achieved in any lab with a minimum cost investment and thus we estimate that this would open a novel area of opportunity on research and development for biomedical applications.

\section{References}

[1] Hisaka M., Sugiura T. and Kawata S., "Optical crosssectional imaging with pulse ultrasound wave assistance", J. Opt. Soc. Am. A., Vol. 18, 2001, pp. $1531-4$.

[2] Quing Zhu and NanGuang Chen, "Imaging tumor angiogenesis by use of combined near-infrared diffusive light and ultrasound", Opt. Lett. Vol. 24, 2003, pp.1050-2.

[3] Arámbula-Cosío F., Lira Berra E., Hevia-Montiel N., García-Segundo C., Garduño E., Alvarado-Gonzáles M., Quispe-Siccha R. M., Reyes-Ramírez B., Hazan-Lasri E., "Computer assisted biopsy of breast tumors", EMBC'10 Review 703, 2010, pp. 5995-5998

[4] Minghua $\mathrm{Xu}$ and Lihong V. Wang, "Photoacoustic imaging in biomedicine", Review of scientific instruments, Vol. 77, 2006, pp.1-22.

[5] Manohar S., Vaatjes S. E., Van Hespen Y.C.G., Klaase J. M., den Engh F. M. V., Steenbergen W., and Van Leeuwen Ton G., "Initial results of in vivo noninvasive cancer imaging in the human breast using nearinfrared photoacoustic", J. Op. Soc. Am. A., Vol. 15, 2007, pp. 12277-12285.

[6] Alexander A. Oraevsky, Steven L. Jacques, and Frank K. Tittel, "Measurement of tissue optical properties by time-resolved detection of laser-induced transient stress", Applied Optic, Vol. 36, 1997, pp. 402-415.

[7] Egerev S., Ermilov S., Ovchinnikov O., Fokin A., Guzatov Dmitry, Klimov V., Kanavin A. and Oraevsky A., "Acoustic signals generated by laser-irradiated metal nanoparticles", Applied Optics, Vol. 48, 2009, pp. 38-45.

[8] Lal S, Clare S. E. and Halas N. J., "Nanoshell-enabled photothermal cáncer therapy: impending clinical impact", Accounts of Chemical Research, Vol. 41(12), 2008, pp.1842-51.

[9] Vilca-Quispe L., Alvarado-Gil J. J., Quintana P., Ordonez-Miranda J., " Diffusion of Methylene Blue in Phantoms of Agar Using a Photoacoustic Technique", Int. J, Thernofisic, Vol. 31, 2010, pp.987-97

[10] Kharine A., Manohar S., Seeton R., Kolkman R. G M, Bolt R. A, Steenbergen W. and de Mu F.F.M., "Poly(vinyl alcohol) gels for use as tissue phantoms in photoacoustic mammography", Phys. Med. Biol. Vol. 48, 2003, pp.1-14.

[11] Fromageau J., Gennisson J.L., Schmitt C., Maurice R.L., Mongrain R., and Cloutier G. "Estimation of 
Polyvinyl Alcohol Cryogel Mechanical Properties with Four Ultrasound Elastography Methods and Comparison with Gold Standard Testings", IEEE Trans. Ultrasonics, Ferroelectrics, and Frequency Control, Vol. 54, 2007, pp. 498-509.

[12] Sato-Berrú Robert, Saniger José M., Flores-Flores José, Sanchez-Espíndola María, "Simple method for the controlled growth of $\mathrm{SiO} 2$ spheres" (private communication in preparation for submission).

[13] http://www.ultrasound-

images.com/breast.htm\#Carcinoma_of_breast.

\section{Aknowledgements}

The authors want to acknowledge this work was made possible thanks the research sponsorships from the Insituto de Ciencia y Tecnología at Mexico City through a grant contract between them and CCADET-UNAM in Breast Cancer Research. 